\title{
ENERGY-DEPENDENT POTENTIALS REVISITED: A UNIVERSAL HIERARCHY OF HYDRODYNAMIC TYPE *
}

\author{
L. Martínez Alonso ${ }^{1}$ and A. B. Shabat ${ }^{2}$ \\ ${ }^{1}$ Departamento de Física Teórica II, Universidad Complutense \\ E28040 Madrid, Spain \\ ${ }^{2}$ Landau Institute for Theoretical Physics \\ RAS, Moscow 117 334, Russia
}

\begin{abstract}
A hierarchy of infinite-dimensional systems of hydrodynamic type is considered and a general scheme for classifying its reductions is provided. Wide families of integrable systems including, in particular, those associated with energy-dependent spectral problems of Schrödinger type, are characterized as reductions of this hierarchy. $N$-phase type reductions and their corresponding Dubrovin equations are analyzed. A symmetry transformation connecting different classes of reductions is formulated.
\end{abstract}

Key words: Integrable systems of hydrodynamic type. Energydependent Schrödinger spectral problems.

1991 MSC: 58B20.

*Partially supported by CICYT proyecto PB98-0821 


\section{Introduction}

In this work we consider the system of evolution equations

$$
\frac{\partial Y}{\partial t_{i}}=\left\langle A_{i}, Y\right\rangle, \quad A_{i}:=\left(\lambda^{i} Y\right)_{+}, \quad i \geq 0
$$

where $\langle U, V\rangle:=U V_{x}-U_{x} V$, and the function

$$
Y=Y(\lambda, \boldsymbol{t}), \quad \boldsymbol{t}:=\left(t_{0}:=x, t_{1}, \ldots\right),
$$

is assumed to admit an expansion

$$
Y=1+\frac{y_{1}(\boldsymbol{t})}{\lambda}+\frac{y_{2}(\boldsymbol{t})}{\lambda^{2}}+\cdots, \quad \lambda \rightarrow \infty .
$$

Here and henceforth we will denote by $A=A(\lambda)_{+}+A(\lambda)_{-}$the standard decomposition of a power series of $\lambda$ in its components $A_{+}$and $A_{-}$with positive and strictly negative powers of $\lambda$, respectively. The system of equations (1) originated in the theory of the finite-gap solutions of the KdV equation (see [1]) and, in a more general context, it appears [2] in the analysis of the integrable hierarchies $\operatorname{EDP}(d)(d \geq 1)$ associated with Schrödinger spectral problems with energy-dependent potentials [3]- [9]

$$
\partial_{x x} \psi=U(\lambda, x) \psi, \quad U(\lambda, x):=\lambda^{d}+\sum_{i=0}^{d-1} \lambda^{i} u_{i}(x) .
$$

The nonlinear evolution equations of the $\operatorname{EDP}(d)$ hierarchy can be written as

$$
\partial_{i} U=-\frac{1}{2} A_{i, x x x}+2 U A_{i, x}+U_{x} A_{i}, \quad A_{i}=\left(\lambda^{i} Y\right)_{+},
$$

where the function $Y$ can be determined by

$$
Y=2 \lambda^{\frac{d}{2}} \frac{\psi_{1}(\lambda) \psi_{2}(\lambda)}{\left\langle\psi_{1}, \psi_{2}\right\rangle}
$$

with $\psi_{1}, \psi_{2}$ being two independent solutions of (2). As a consequence of (2) and (4) it follows that

$$
2 Y_{x x} Y-Y_{x}^{2}-4 U Y^{2}+4 \lambda^{d}=0 .
$$


This equation allows us to determine the coefficients of $Y$ as differential polynomials depending on the potential coefficients $\left\{u_{i}=u_{i}(x)\right\}_{i=0}^{d-1}$. In this way, the solutions of (11) provided by the $\operatorname{EDP}(d)$ hierarchy satisfy a reduction condition described by the differential constraint (5). For example

i) In the case of the $\operatorname{EDP}(1)$ hierarchy, the standard KdV hierarchy, we have $U=\lambda+u_{0}$ and

$$
\begin{gathered}
u_{0}=-2 y_{1}, y_{2}=\frac{3}{2} y_{1}^{2}+\frac{1}{4} y_{1, x x} \\
8 y_{3}=8 y_{1}^{3}-\left(y_{1, x}\right)^{2}+2 y_{1} y_{1, x x}+8 y_{1} y_{2}+2 y_{2, x x} .
\end{gathered}
$$

ii) The hierarchy $\operatorname{EDP}(2)$ is equivalent to the Zakharov-Shabat hierarchy [3] and one finds

$$
u_{1}=-2 y_{1}, u_{0}=3 y_{1}^{2}-2 y_{2}, \quad y_{3}=12 y_{1} y_{2}-8 y_{1}^{3}+y_{1, x x} .
$$

iii) For the case $\operatorname{EDP}(3)$ the first few relations arising from (5) are

$$
u_{2}=-2 y_{1}, u_{1}=3 y_{1}^{2}-2 y_{2}, u_{0}=-4 y_{1}^{3}+6 y_{1} y_{2}-2 y_{3} .
$$

The purpose of this paper is to study (11) as a hierarchy of nonlinear integrable models. In this sense, the form of (四) resembles that of the dispersionless KP hierarchy [10]-16]

$$
\frac{\partial Z}{\partial t_{i}}=\left\{B_{i}, Z\right\}, \quad B_{i}:=\left(Z^{i}\right)_{+}, \quad i \geq 0
$$

where $\{U, V\}:=U_{\lambda} V_{x}-U_{x} V_{\lambda}$ is the standard Poisson bracket operation and $Z=Z(\lambda, \boldsymbol{t})$ is assumed to admit an expansion

$$
Z=\lambda+\frac{z_{1}(\boldsymbol{t})}{\lambda}+\frac{z_{2}(\boldsymbol{t})}{\lambda^{2}}+\cdots, \quad \lambda \rightarrow \infty .
$$

The subsequent analysis proves that the hierarchy (1), like the dispersionless KP hierarchy, has deep connections with the theory of hydrodynamic systems. In fact, we notice that, in terms of the coefficients of the expansion of $Y$, the hierarchy (四) becomes a set of infinite-dimensional systems of hydrodynamic type

$$
\partial_{i} y_{n}=\sum_{k=1}^{n}\left\langle y_{n-k}, y_{i+k}\right\rangle, \quad n \geq 1
$$


We will show that the hierarchy (1) exhibits a rich reduction theory which includes not only the standard types of reductions of the dispersionless KP hierarchy but also reductions of differential type which contain, in particular, the integrable hierarchies $\operatorname{EDP}(d)$. From this point of view the hierarchy (1) manifests a universal character.

It should be observed that the zeros $\lambda=\gamma$ of $Y$ are Riemann invariants for (1) as they satisfy

$$
\frac{\partial \gamma}{\partial t_{i}}=A_{i}(\gamma) \partial_{x} \gamma
$$

This fact allows one to write the dynamical equations of wide families of reductions in Riemann invariant form. In the case of differential reductions, the systems (10) have to be enlarged with appropriate equations of Dubrovin type [1].

One of the results of our work is the introduction of a symmetry transformation for (11) which connects different classes of reductions. In particular, it transforms every $\operatorname{EDP}(d)$ hierarchy into its corresponding $\operatorname{EDP}(d+2)$ hierarchy.

\section{Reductions, $N$-phase solutions and Dubrovin's equations}

\subsection{General scheme}

Many interesting reductions of the hierarchy (1) can be formulated. We will be here concerned with those determined by constraints of the form

$$
F\left(\lambda, Y_{0}, Y_{1}, \ldots, Y_{n}\right)_{-}=0, \quad Y_{j}:=\partial_{x}^{j} Y
$$

where it is assumed that

$$
\frac{\partial F}{\partial Y_{n}} \neq 0
$$

Reductions of this type will be henceforth referred to as differential reductions of order $n$. The condition for (11) to determine one of such reductions is that for all $i \geq 1$ the function $F$ must satisfy

$$
\frac{\mathrm{d} F_{-}}{\mathrm{d} t_{i}}=\left(\sum_{j=0}^{n} F_{j} \partial_{i} Y_{j}\right)_{-}=0, \quad F_{j}:=\frac{\partial F}{\partial Y_{j}},
$$


provided $Y$ verifies (11) and (11). By taking into account that (11) implies

$$
\left(\sum_{j=0}^{n} F_{j} Y_{j+1}\right)_{-}=0
$$

it follows that (12) is equivalent to

$$
\left(\sum_{j=0}^{n} F_{j} Y \partial_{x}^{j+1} A_{i}-\sum_{j=1}^{n}\left(\sum_{k=j}^{n} c_{k j} F_{k} Y_{k+1-j}\right) \partial_{x}^{j} A_{i}\right)_{-}=0
$$

where

$$
c_{k j}=\left(\begin{array}{c}
k \\
j
\end{array}\right)-\left(\begin{array}{c}
k \\
j-1
\end{array}\right) .
$$

Thus, it is obvious that a sufficient condition for (13) to hold is that $F$ satisfies a system of partial differential equations of the form

$$
\begin{gathered}
F_{j-1}-\sum_{k=j}^{n} c_{k j} F_{k} Y_{k+1-j}=\alpha_{j} F+\beta_{j}, \quad j=1, \ldots, n \\
F_{n} Y=\alpha_{n+1} F+\beta_{n+1},
\end{gathered}
$$

for a given set $\left\{\alpha_{j}, \beta_{j}\right\}_{j=1}^{n+1}$ of entire functions of $\lambda$ (i.e. $\left.\left(\alpha_{j}\right)_{-}=\left(\beta_{j}\right)_{-}=0\right)$.

\subsection{Differential reductions of order 0}

According to the general scheme, reductions of the form

$$
F(\lambda, Y)_{-}=0
$$

can be generated by solving

$$
Y \frac{\partial F}{\partial Y}=\alpha F+\beta
$$

for given functions $\alpha(\lambda)$ and $\beta(\lambda)$ satisfying $\alpha_{-}=\beta_{-}=0$. The general solution of (16) is

$$
\begin{gathered}
\alpha \equiv 0, \quad F=\beta(\lambda) \ln Y+a(\lambda), \\
\alpha \neq 0, \quad F=a(\lambda) Y^{\alpha(\lambda)}-\frac{\beta(\lambda)}{\alpha(\lambda)},
\end{gathered}
$$


where $a(\lambda)$ is an arbitrary function of $\lambda$.

One of the simplest types of reductions (15) included in the class (17) is

$$
\left(\lambda^{N} Y\right)_{-}=0, \quad N \geq 1,
$$

which means that $Y$ is of the form

$$
Y=1+\frac{y_{1}(\boldsymbol{t})}{\lambda}+\cdots+\frac{y_{N}(\boldsymbol{t})}{\lambda^{N}} .
$$

We will refer to the reduction (19) as the $N$-phase reduction of (1). Under the

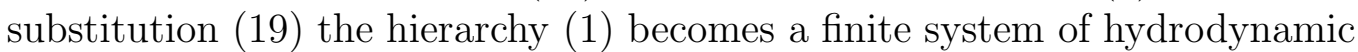
type for the coefficients $\left\{y_{i}\right\}_{i=1}^{N}$.

\section{Example}

For 2-phase reductions

$$
Y=1+\frac{y_{1}}{\lambda}+\frac{y_{2}}{\lambda^{2}},
$$

the system (11) becomes

$$
\begin{gathered}
\partial_{1} y_{1}=\partial_{x} y_{2}, \quad \partial_{1} y_{2}=\left\langle y_{1}, y_{2}\right\rangle, \\
\partial_{n} y_{1}=\partial_{n} y_{2}=0, \quad n \geq 2 .
\end{gathered}
$$

Thus, by introducing a function $Z$ such that

$$
y_{1}=Z_{x}, \quad y_{2}=Z_{t}, \quad t:=t_{1},
$$

the 2-phase reductions of (1) are determined by the solutions of the homogeneous Monge-Ampere equation

$$
Z_{t t}=Z_{x} Z_{t x}-Z_{t} Z_{x x}
$$

which in turn reduces to the linear equation

$$
W_{p p}+p W_{p q}+q W_{q q}=0,
$$

under the Legendre transformation $(x, t) \mapsto(p, q)$

$$
W=x p+t q-Z, \quad p=Z_{x}, \quad q=Z_{t} .
$$


It should be noticed that an inhomogeneous version of the Monge-Ampere equation (22) describes the class of reductions of the dispersionless KP hierarchy which depend on two independent functions [14]-16].

The $N$-phase reductions admit a complete set of Riemann invariants supplied by the zeros $\left\{\gamma_{i}\right\}_{i=1}^{N}$ of the function $Y$

$$
Y=\frac{1}{\lambda^{N}} \prod_{i=1}^{N}\left(\lambda-\gamma_{i}\right) .
$$

Thus, under the change of dependent variables

$$
\boldsymbol{y}=\left(y_{1}, \ldots, y_{N}\right) \mapsto \boldsymbol{\gamma}=\left(\gamma_{1}, \ldots, \gamma_{N}\right),
$$

the system (11) can be written as

$$
\partial_{i} \gamma=\beta_{i}(\gamma) \partial_{x} \gamma, \quad i \geq 0
$$

Here $\beta_{i}$ are the diagonal matrices

$$
\left(\beta_{i}\right)_{j k}=A_{i}\left(\gamma_{j}\right) \delta_{j k}=\left(\gamma_{j}^{i}+\gamma_{j}^{i-1} y_{1}(\gamma)+\cdots+y_{i}(\gamma)\right) \delta_{j k}
$$

the functions $y_{n}(\gamma)$ are given by the symmetric polynomials

$$
y_{n}(\gamma)=(-1)^{n} \sum_{I} \gamma_{i_{1}} \cdots \gamma_{i_{n}}
$$

and the sum extends to all subsets $I \subset\{1, \ldots, N\}$ with $n$ elements. Equations (23) are well known in the theory of $N$-phase solutions of the $\mathrm{KdV}$ hierarchy [17].

\subsection{Differential reductions of order 1}

Reductions of the form

$$
F\left(\lambda, Y, Y_{x}\right)_{-}=0
$$

can be determined by solving

$$
Y \frac{\partial F}{\partial Y}=\alpha_{1} F+\beta_{1}, \quad Y \frac{\partial F}{\partial Y_{x}}=\alpha_{2} F+\beta_{2},
$$


for given functions $\alpha_{i}, \beta_{i}$ with $\left(\alpha_{i}\right)_{-}=\left(\beta_{i}\right)_{-}=0$. The compatibility conditions for these equations imply

$$
\alpha_{1}=-1, \quad \alpha_{2}=0,
$$

so that the general solution of the system (25) is

$$
F=\alpha(\lambda) \frac{Y_{x}}{Y}+\frac{a(\lambda)}{Y}+\gamma(\lambda)
$$

where $a$ is an arbitrary function of $\lambda$, and we are denoting $\alpha(\lambda):=\beta_{2}(\lambda), \gamma(\lambda):=$ $\beta_{1}(\lambda)$. For a function $F$ of the form (26) the reduction constraint (24) reads

$$
\alpha(\lambda) Y_{x}+a(\lambda)=\beta(\lambda, \boldsymbol{t}) Y
$$

where

$$
\beta(\lambda, \boldsymbol{t}):=\left(\alpha(\lambda) \frac{Y_{x}}{Y}+\frac{a(\lambda)}{Y}\right)_{+}
$$

\section{Examples}

1) The reduction corresponding to (26) with

$$
F=\alpha \frac{Y_{x}}{Y}+\frac{\lambda}{Y}
$$

where $\alpha$ is a given nonzero complex number, is determined by

$$
\alpha Y_{x}+\lambda=\left(\lambda-y_{1}\right) Y
$$

or, equivalently, by the following recurrence relation for the coefficients of the expansion of $Y$

$$
y_{i+1}=y_{1} y_{i}+\alpha \partial_{x} y_{i}, \quad i \geq 0
$$

2) If we set

$$
F=\alpha \frac{Y_{x}}{Y}+\frac{\lambda^{2}}{Y}
$$

where $\alpha$ is a given nonzero complex number, then the reduction constraint (27) can be written as

$$
\alpha Y_{x}+\lambda^{2}=\left(\lambda^{2}-\lambda y_{1}+y_{1}^{2}-y_{2}\right) Y
$$


which imposes the following recurrence relation

$$
y_{i+2}=y_{1} y_{i+1}+\left(y_{2}-y_{1}^{2}\right) y_{i}+\alpha \partial_{x} y_{i}, \quad i \geq 0 .
$$

Under appropriate conditions it is possible to formulate combinations of both $N$-phase and differential reductions. The coefficients $\left\{y_{n}\right\}_{n=1}^{N}$ of the corresponding function $Y$ are constrained by a certain system of differential equations with respect to the $x$ variable. In terms of the zeros $\left\{\gamma_{i}\right\}_{i=1}^{N}$ of $Y$ these differential equations are of Dubrovin type [1].

For example, from (27) it follows that the condition for differential reductions of order 1 to admit a further $N$-phase reduction is that the function

$$
\Phi:=\lambda^{N} Y=\prod_{i=1}^{N}\left(\lambda-\gamma_{i}\right)
$$

satisfies

$$
\alpha(\lambda) \Phi_{x}+\lambda^{N} a(\lambda)=\beta(\lambda, \boldsymbol{t}) \Phi .
$$

This condition can be fulfilled only if $\left(\lambda^{N} a\right)_{-}=0$. Furthermore, by inserting (34) into (35) we get the following system of Dubrovin's equations

$$
\partial_{x} \gamma_{i}=\frac{\gamma_{i}^{N} a\left(\gamma_{i}\right)}{\alpha\left(\gamma_{i}\right) \prod_{j \neq i}\left(\gamma_{i}-\gamma_{j}\right)}, \quad i=1, \ldots, N
$$

\section{Examples}

1) From (30) we have that the 2-phase reduction of the differential reduction (29) is determined by

$$
y_{2}=y_{1}^{2}+\alpha \partial_{x} y_{1}, \quad y_{n}=0, \quad n \geq 3,
$$

where $y_{1}$ verifies the differential equation

$$
\alpha^{2} \partial_{x x} y_{1}+3 \alpha y_{1} \partial_{x} y_{1}+y_{1}^{3}=0 \text {. }
$$

Let $\left\{\gamma_{i}\right\}_{i=1}^{2}$ be the zeros of $Y$, then we can write $y_{1}=\gamma_{1}+\gamma_{2}, y_{2}=\gamma_{1} \gamma_{2}$. Moreover, (37) and (38) lead to the Dubrovin's equations

$$
\alpha \partial_{x} \gamma_{i}=\frac{\gamma_{i}^{3}}{\prod_{j \neq i}\left(\gamma_{i}-\gamma_{j}\right)}, \quad i=1,2 .
$$


We observe that this reduction describes the 2-phase solutions of Burgers equation. Indeed, according to (21) and (37) it follows

$$
u_{t}=\alpha u_{x x}+2 u u_{x}, \quad u:=y_{1}, t:=t_{1} .
$$

2) Equation (33) implies that the 2-phase reduction satisfying the condition (32) is characterized by

$$
y_{2}=\frac{1}{2}\left(y_{1}^{2}-\alpha \frac{\partial_{x} y_{1}}{y_{1}}\right), \quad y_{n}=0, \quad n \geq 3
$$

where $y_{1}$ verifies

$$
-2 \alpha^{2} y_{1} \partial_{x x} y_{1}+4 \alpha y_{1}^{3} \partial_{x} y_{1}+3 \alpha^{2}\left(\partial_{x} y_{1}\right)^{2}-y_{1}^{3}=0 .
$$

These equations lead to the Dubrovin's equations

$$
\alpha \partial_{x} \gamma_{i}=\frac{\gamma_{i}^{4}}{\prod_{j \neq i}\left(\gamma_{i}-\gamma_{j}\right)}, \quad i=1,2
$$

From (21) and (41) we have that this reduction represents the 2-phase solutions of the evolution equation

$$
u_{t}=-\frac{1}{2} \partial_{x}\left(\alpha \frac{u_{x}}{u}-u^{2}\right), \quad u:=y_{1}, t:=t_{1} .
$$

\subsection{Differential reductions of order 2}

Let us consider now differential reductions of second order

$$
F\left(\lambda, Y, Y_{x}, Y_{x x}\right)_{-}=0
$$

They are characterized by the solutions of

$$
\begin{gathered}
Y \frac{\partial F}{\partial Y}-Y_{x x} \frac{\partial F}{\partial Y_{x x}}=\alpha_{1} F+\beta_{1}, \\
Y \frac{\partial F}{\partial Y_{x}}+Y_{x} \frac{\partial F}{\partial Y_{x x}}=\alpha_{2} F+\beta_{2}, \\
Y \frac{\partial F}{\partial Y_{x x}}=\alpha_{3} F+\beta_{3},
\end{gathered}
$$


for given functions $\alpha_{i}(\lambda), \beta_{i}(\lambda)$ with $\left(\alpha_{i}\right)_{-}=\left(\beta_{i}\right)_{-}=0$. Equivalently, these conditions can be expressed as

$$
\begin{gathered}
\frac{\partial F}{\partial Y}=\frac{\alpha_{1} F+\beta_{1}}{Y}+\frac{Y_{x x}}{Y^{2}}\left(\alpha_{3} F+\beta_{3}\right), \\
\frac{\partial F}{\partial Y_{x}}=\frac{\alpha_{2} F+\beta_{2}}{Y}-\frac{Y_{x}}{Y}\left(\alpha_{3} F+\beta_{3}\right), \\
\frac{\partial F}{\partial Y_{x x}}=\frac{\alpha_{3} F+\beta_{3}}{Y} .
\end{gathered}
$$

The compatibility conditions for these equations imply

$$
\alpha_{1}=-2, \quad \alpha_{2}=\alpha_{3}=\beta_{2}=0,
$$

and the following general solution of (46) arises

$$
F=\alpha(\lambda)\left(\frac{Y_{x x}}{Y}-\frac{1}{2} \frac{Y_{x}^{2}}{Y^{2}}\right)+\frac{a(\lambda)}{Y^{2}}+\beta(\lambda)
$$

where $a=a(\lambda)$ is an arbitrary function and $\alpha(\lambda):=\beta_{3}(\lambda), \beta(\lambda):=-\beta_{3}(\lambda) / 2$. If we set

$$
\alpha(\lambda)=2, \quad a(\lambda)=4 \lambda^{d}
$$

in (47), it follows that the corresponding reductions are the hierarchies $\operatorname{EDP}(d)$ associated to the Schrödinger spectral problems with energy-dependent potentials (2)-(3). Indeed it is enough to observe that (47) and (48) determine a reduction characterized by an equation of the form

$$
2 Y_{x x} Y-Y_{x}^{2}-4 U Y^{2}+4 \lambda^{d}=0
$$

where $U$ is a polynomial in $\lambda$

$$
U(\lambda, \boldsymbol{t}):=\lambda^{d}+\sum_{i=0}^{d-1} \lambda^{i} u_{i}(\boldsymbol{t}),
$$

the coefficients of which can be recursively found from those of $Y$ trough (49).

From (49) it is clear that the $\operatorname{EDP}(d)$ hierarchies admit $N$-phase reductions. If we introduce

$$
\Phi:=\lambda^{N} Y=\prod_{i=1}^{N}\left(\lambda-\gamma_{i}\right)
$$


then (49) reads

$$
2 \Phi_{x x} \Phi-\Phi_{x}^{2}-4 U \Phi^{2}+4 \lambda^{d+2 N}=0
$$

which is equivalent to the following system of Dubrovin's equations

$$
\left(\partial_{x} \gamma_{i}\right)^{2}=\frac{4 \gamma_{i}^{d+2 N}}{\prod_{j \neq i}\left(\gamma_{i}-\gamma_{j}\right)^{2}}, \quad i=1, \ldots, N
$$

\section{$3 \quad$ Symmetry transformations}

The hierarchy (1) exhibits an interesting type of symmetry transformations. Let

$$
Y=1+\frac{y_{1}(\boldsymbol{t})}{\lambda}+\frac{y_{2}(\boldsymbol{t})}{\lambda^{2}}+\ldots
$$

be a solution of (11), and take a solution $X=X\left(t_{1}, t_{2}, \ldots\right)$ of the associated system of differential equations

$$
\partial_{i} X+y_{i}\left(X, t_{1}, t_{2}, \ldots\right)=0, \quad i \geq 1
$$

then it follows that the function

$$
Y^{\prime}\left(\lambda, \boldsymbol{t}^{\prime}\right):=Y\left(\lambda, \boldsymbol{t}\left(\boldsymbol{t}^{\prime}\right)\right)
$$

where $\boldsymbol{t}^{\prime}:=\left(x^{\prime}, t_{1}^{\prime}, t_{2}^{\prime}, \ldots, t_{n}^{\prime}, \ldots\right)$ and

$$
\boldsymbol{t}\left(\boldsymbol{t}^{\prime}\right):=\left(X\left(x^{\prime}, t_{1}^{\prime}, \ldots\right), x^{\prime}, t_{1}^{\prime}, \ldots, t_{n-1}^{\prime}, \ldots\right),
$$

is also a solution of (11). In order to prove this property we notice that from (1) it follows at once that

$$
\partial_{i} A_{j}-\partial_{j} A_{i}=\left\langle A_{i}, A_{j}\right\rangle, \quad A_{i}:=\left(\lambda^{i} Y\right)_{+},
$$

which means that (11) defines a family of commuting vector fields in the space of Laurent series of $\lambda$ with coefficients depending on $\left\{y_{i}\right\}_{i \geq 1}$. By setting $\lambda=0$ in (57) we find

$$
\partial_{i} y_{j}-\partial_{j} y_{j}=\left\langle y_{i}, y_{j}\right\rangle
$$


Hence, the contracted vector fields

$$
\mathcal{D}_{i}:=\partial_{i}-y_{i} \partial_{x}, \quad i \geq 1,
$$

form a commutative family as well. In this way, given a solution $Y=Y(\lambda, \boldsymbol{t})$ of (11) then one has

$$
\mathcal{D}_{i} Y=\left\langle\left(\lambda^{i-1} Y\right)_{+}, Y\right\rangle_{1}, \quad i \geq 1,
$$

where $\langle A, B\rangle_{1}:=A \partial_{1} B-B \partial_{1} A$. Therefore, it readily follows that $Y^{\prime}$ is also a solution of (1) and, consequently, the transformation $Y \rightarrow Y^{\prime}$ is a symmetry of the hierarchy (1).

\section{Example}

An elementary solution of (22) is given by

$$
Z=-\frac{a}{b} e^{-b t}+\frac{b}{2} x^{2} .
$$

It determines the following 2-phase solution of (1)

$$
Y=1+\frac{b x}{\lambda}+\frac{a e^{-b t_{1}}}{\lambda^{2}} .
$$

The associated system (54) is

$$
\frac{\partial X}{\partial t_{1}}+b X=0, \quad \frac{\partial X}{\partial t_{2}}+a e^{-b t_{1}}=0,
$$

which has the solution

$$
X=\left(c-a t_{2}\right) e^{b t_{1}} .
$$

Hence, from (55) we get a new solution of (11)

$$
Y=1+\frac{b\left(c-a t_{1}\right) e^{-b x}}{\lambda}+\frac{a e^{-b x}}{\lambda^{2}} .
$$

An interesting aspect of the transformation $Y \rightarrow Y^{\prime}$ is that it establishes some relationships between different differential reductions of (1). We notice that (54) and (55) imply

$$
\begin{gathered}
Y_{x}=\frac{1}{\lambda}\left(\partial_{x^{\prime}} Y^{\prime}+\partial_{x} y_{1} Y^{\prime}\right), \\
Y_{x x}=\frac{1}{\lambda^{2}}\left(Y_{x^{\prime} x^{\prime}}^{\prime}+\partial_{x} y_{1} Y_{x^{\prime}}^{\prime}+\left(\lambda \partial_{x x} y_{1}+\partial_{x x} y_{2}-y_{1} \partial_{x x} y_{1}\right) Y^{\prime}\right),
\end{gathered}
$$


These formulas are useful to analyze the transformation properties of the differential reductions under $Y \rightarrow Y^{\prime}$. For example, if $Y$ satisfies a differential reduction (27) of order 1

$$
\alpha(\lambda) Y_{x}+a(\lambda)=\beta(\lambda, \boldsymbol{t}) Y
$$

then $Y^{\prime}$ satisfies a similar differential reduction

$$
\alpha(\lambda) Y_{x^{\prime}}^{\prime}+\lambda a(\lambda)=\left(\lambda \beta\left(\lambda, \boldsymbol{t}\left(\boldsymbol{t}^{\prime}\right)\right)-\alpha(\lambda) \partial_{x} y_{1}\right) Y^{\prime}
$$

The way in which $\operatorname{EDP}(d)$ reductions transform under (54)-(55) is particularly simple. Indeed, let us suppose that $Y$ satisfies

$$
2 Y_{x x} Y-Y_{x}^{2}-4 U Y^{2}+4 \lambda^{d}=0, \quad U(\lambda, x):=\lambda^{d}+\sum_{i=0}^{d-1} \lambda^{i} u_{i}(x)
$$

then, as a consequence of $(60)$, the transformed function $Y^{\prime}$ verifies

$$
2 Y_{x^{\prime} x^{\prime}}^{\prime} Y^{\prime}-\left(Y_{x^{\prime}}^{\prime}\right)^{2}-4 U^{\prime} Y^{\prime 2}+4 \lambda^{d+2}=0
$$

where

$$
U^{\prime}:=\lambda^{2} U-\frac{1}{2}\left(\lambda \partial_{x x} y_{1}+\partial_{x x} y_{2}-y_{1} \partial_{x x} y_{1}\right)+\frac{1}{4}\left(\partial_{x} y_{1}\right)^{2}
$$

This means that $Y \rightarrow Y^{\prime}$ transforms the $\operatorname{EDP}(d)$ hierarchy into the $\operatorname{EDP}(d+$ 2) hierarchy. In particular, this result proves that the whole family of hierarchies of integrable models associated with energy-dependent Schrödinger problems can be generated from its two first members, namely: the KdV hierarchy and the Zakharov-Shabat hierarchy.

From (60) one can also derive the transformation properties of Dubrovin's equations under the symmetry $Y \rightarrow Y^{\prime}$. Obviously, if $Y$ satisfies a $N$-phase reduction so does $Y^{\prime}$. Moreover, the zeros of $Y$ transform as $\gamma_{i}^{\prime}\left(\boldsymbol{t}^{\prime}\right)=\gamma_{i}\left(\boldsymbol{t}\left(\boldsymbol{t}^{\prime}\right)\right)$ and (60) lead to identities of the type $\partial_{x^{\prime}} \gamma_{i}^{\prime}=\gamma_{i}^{\prime} \partial_{x} \gamma_{i}$. 


\section{Acknowledgements}

A.B. Shabat was supported by the Russian Foundation for Basic Research (Grant Nos. 96-15-96093 and 98-01-01161), INTAS (Grant No. 99-1782) and a Rotschild professorship. L. Martinez Alonso was supported by the Fundación Banco Bilbao Vizcaya Argentaria. Both authors wish to thank

the organizers of the program Integrable Systems at the Newton Institute of Cambridge University for their warm hospitality.

\section{References}

[1] B. A. Dubrovin, V. B. Matveev and S. P. Novikov, Usp. Mat. Nauk 31, No. 1, 55 (1976).

[2] V. G. Mikhalev, Funct. Anal. Appl. 26 No 2, 140 (1992).

[3] M. Jaulent and I. Miodek, Lett. Math. Phys. 1, 243 (1976); Lett. Nuovo Cimento 20, 655 (1977).

[4] L. Martínez Alonso, J. Math. Phys. 21, 2342 (1980).

[5] M. Antonowicz and A. P. Fordy, Physica 28 D, 345 (1987).

[6] M. Mañas, L. Martínez Alonso and E. Medina, J.Phys. A: Math. Gen. 30, 4815 (1997).

[7] A. N. W. Hone, Phys. Lett. A 249, 46 (1998).

[8] B. Konopelchenko, L. Martínez Alonso and E. Medina, J.Phys. A: Math. Gen. 32, 3621 (1999).

[9] A. B. Shabat, Theor. Math. Phys. 121 1, 1397 (1999).

[10] I.M.Krichever, Commun. Pure. Appl. Math. 47, 437 (1992); Commun. Math. Phys. 143, 415 (1992).

[11] T.Takasaky and T.Takebe, Int. J. Mod. Phys. A 7 Suppl. 1B, 889 (1992); Rev. Math. Phys. 7, 743 (1995).

[12] Y.Kodama, Phys.Lett. A 129, 223 (1988). 
[13] Y.Kodama and J.Gibbons, Phys. Lett. A 135, 167 (1989).

[14] J.Gibbons and S.P.Tsarev, Phys. Lett. A 211, 19 (1996).

[15] J.Gibbons and S.P.Tsarev, Phys. Lett. A 258, 263 (1999).

[16] M. Mañas, L. Martinez Alonso and E. Medina, J. Phys. A: Math. Gen. 35, 401 (2002)

[17] E.V. Ferapontov, Phys. Lett. A 158, 112 (1991). 